\title{
COPD Exacerbation History and Impact on Future Exacerbations - 8-Year Retrospective Observational Database Cohort Study from Germany
}

\author{
Claus F Vogelmeier' \\ Joanna Diesing ${ }^{2}$ \\ Nils Kossack ${ }^{2}$ \\ Marc Pignot ${ }^{3}$ \\ Felix W Friedrich (D) ${ }^{4}$ \\ 'Department of Medicine, Pulmonary and \\ Critical Care Medicine, University \\ Medical Center Giessen and Marburg, \\ Philipps-Universität Marburg, Marburg, \\ Hessen, Germany; ${ }^{2}$ WIG2 GmbH \\ Scientific Institute for Health Economics \\ and Health System Research, Leipzig, \\ Sachsen, Germany; ${ }^{3}$ Kantar Health, \\ Munich, Germany; ${ }^{4}$ AstraZeneca, Wedel, \\ Germany
}

Background: Recent studies evaluating the predictive value of different variables on future exacerbations suggest exacerbation history as the strongest predictor. We examined the effect of exacerbation history on subsequent events in a large sample population with over 250,000 COPD patients using up to 8 years of longitudinal healthcare data from Germany.

Methods: Patients 40 years or older with any COPD diagnosis in primary or secondary care were included from 2011 to 2017 (index period) from healthcare insurance claims (Germany; WIG2 research database), with 12 months before index date as baseline and at least 12month follow-up. Exacerbations during baseline were defined as moderate (treatment with oral corticosteroids or antibiotics, J01AA, J01CA) or severe (emergency visit or hospitalization).

Results: Patients without (category A), with one moderate (category B), or with either one severe or several baseline exacerbations (category $C$ ) experienced an average of 0.9 (CI 0.9 0.9), 1.9 (CI 1.9-1.9), and 6.3 (CI 6.1-6.3) exacerbations during the first 3 years of followup, respectively. By 8 years, 87.0\% (CI 86.6-87.4), 70.5\% (CI 69.9-71.0) and 49.1\% (CI 48.9-49.3) of category C, B and A patients had experienced a subsequent exacerbation.

Conclusion: Baseline exacerbations increased the likelihood of, and reduced time to subsequent exacerbations. Even patients without baseline exacerbations experienced exacerbations within three years, emphasizing the importance of adequate treatment in patients with less severe disease presentation as well.

Keywords: chronic obstructive pulmonary disease, COPD, exacerbations, economic burden, healthcare resource utilization

\section{Introduction}

Chronic obstructive pulmonary disease (COPD) is characterized by persistent respiratory symptoms and usually progressive airflow limitation that is not fully reversible. COPD was the third leading cause of death worldwide in $2019,{ }^{1}$ but is both preventable and treatable. COPD is diagnosed by spirometry, with a postbronchodilator FEV1/FVC $<0.70$ confirming persistent airflow limitation. ${ }^{2}$ A recent review by Han et $\mathrm{al}^{3}$ presented evidence for significant airway damage in individuals with respiratory symptoms, even long before airflow obstruction can be spirometrically confirmed. In Europe, premature death and disability induced by COPD costs nearly $€ 50$ billion annually (of both direct and indirect costs); the
Correspondence: Felix W Friedrich AstraZeneca GmbH, Tinsdaler Weg 183 , Wedel, D-22880, Germany

Tel $+49162 \quad 1398835$

Email felix.friedrich@astrazeneca.com 
European Respiratory Society used data from the 2011 WHO Health Statistics and the Global Burden of Disease study to estimate a monetized value of $€ 93$ billion in disability-adjusted life years (DALY) is lost annually in Europe, due to COPD. ${ }^{4}$ Costs associated with exacerbations account for $50-75 \%$ of COPD costs. ${ }^{5,6}$

Exacerbations accelerate disease progression, and patients hospitalized for COPD exacerbations have a poor long-term prognosis, ${ }^{7}$ and a $55 \%$ five-year mortality rate. $^{8}$ As outlined in the 2021 Global Initiative for Chronic Obstructive Lung Disease (GOLD) document, pharmacological therapy attempts to reduce symptoms, frequency, and severity of exacerbations, and improve exercise tolerance and health status. ${ }^{2}$

Recent non-interventional studies using administrative databases, primary care data and other observational data have used models to evaluate the predictive value of several variables on future exacerbations, and exacerbation history seems to be the strongest predictor., ${ }^{9,10}$ Observational studies with similar results are usually restricted to patients on certain treatments, with restrictive inclusion criteria, or only offer results over follow-up periods around 2 years or less. ${ }^{11,12}$ Since healthcare systems cannot always be compared, correlations drawn from other locations cannot reliably be extrapolated to healthcare systems in other countries. There is therefore a need to further understand the risks and costs in the context of the German healthcare system, considering local COPD disease management practices.

This study examined the effect of exacerbation history on subsequent exacerbations in a large sample population with over 250,000 COPD patients, using up to 8 years of longitudinal healthcare data from Germany. Some of these data have been previously presented in conference abstract form. ${ }^{13-16}$

\section{Materials and Methods Study Design}

This is an observational cohort study with cross-sectional and longitudinal analyses of anonymized healthcare claims data of patients diagnosed with COPD in Germany.

\section{Database}

The WIG2 (Scientific Institute for Health Economics and Health System Research) database - an anonymous healthcare claims database with longitudinal data from approximately 4.5 million patients in Germany insured by statutory health insurance (SHI) providers-provides a representative sample (in terms of age, gender, and morbidity) of the German population ${ }^{17}$ on which analyses were done. WIG2 receives the data anonymized, rendering approval by an institutional review board unnecessary (by law). Including baseline and follow-up, data were available from January 1st, 2010 to December 31st, 2018.

Diagnosis data is documented by ICD-10 (International classification of disease, version 10) codes, and drugs were identified by their ATC (Anatomical Therapeutic Chemical classification) code.

\section{Study Population}

Patients 40 years of age or older with any COPD diagnosis (in our database, primary or secondary diagnoses, J44) in primary or secondary care were included in the study index period (January 1st, 2011 to December 31st, 2017), with the first COPD diagnosis in the study index period as their index date. Therefore, all patients, regardless of having a COPD diagnosis before the study index period or not (both incident and non-incident patients), were included in our cohort. Patients had to have a minimum 12-month pre-index data (baseline), and at least 12-months of follow-up data after index date (except for patients deceased within the 12-months follow-up), and there were no other exclusion criteria. A follow-up period of up to 8 years was available for patients with an index date in 2011. Follow-up ended on the date of death, end of insurance coverage, or end of study, whichever occurred first.

\section{Objectives}

The primary goal of the study was to determine the frequency and rate of moderate and severe exacerbations, grouped by severity of disease at baseline according to observed exacerbation history. We also describe treatment patterns at baseline, also by exacerbation history, and both baseline and follow-up healthcare resource use.

\section{Variables}

Data on follow-up time (total, mean, and median, in person years), as well as age (mean and range), gender, and certain comorbidities typically associated with COPD were evaluated during baseline (identified by documentation of diagnosis codes). Data on utilization of inhaled treatments during the baseline period were also collected. Exacerbation type and frequency at baseline were stratified by baseline treatment groups, as defined below. 
Baseline Inhaled Maintenance Respiratory Treatment Patients were stratified into one of six treatment groups by their use of inhaled maintenance treatment (long-acting $\beta 2$-agonist, LABA; long-acting muscarinic antagonist, LAMA; and/or inhaled corticosteroid, ICS) during the baseline period: either LABA or LAMA monotherapy, LABA/LAMA combination therapy, ICS monotherapy, ICS/LABA or ICS/LAMA combination therapy, triple combination of ICS/LABA/LAMA, or none of the above treatments. If the treatment was documented at any time during baseline, it was considered for the baseline treatment groups. The duration and timing of treatments was not considered.

\section{Exacerbations}

We examined both the number and severity of baseline and subsequent exacerbations, using an ATC code for either an oral corticosteroid (OCS) (code $\mathrm{H} 02 \mathrm{AB}$ ) or respiratory antibiotics (codes J01AA or J01CA) to define a moderate exacerbation, or an ICD-10 code (J44) for a COPD-related hospitalization or emergency visit, defining severe exacerbations (see Table S1). Documentation of more than one exacerbation within a 2-week time frame was considered a single exacerbation, with the highest severity exacerbation taking precedence.

We grouped patients into one of three baseline exacerbation categories, based on the 12 months prior to index: no prior exacerbations (A), one moderate prior exacerbation (B), and either one severe or multiple prior exacerbations (regardless of severity) (C).

The percentage of patients by baseline exacerbation category and exacerbation rate in the 12 months before index date (with 95\% confidence intervals, CI) were evaluated. Risk of a first exacerbation during follow-up was determined for each category (adjusted for the risk factors age, gender and comorbidities, using Fine-Gray regression accounting for competing risks of death). ${ }^{18}$ A multivariable Cox proportional hazards model was used to describe the association (by hazard ratios) of baseline exacerbation categories and future moderate and severe exacerbations. Cumulative incidence of a first exacerbation was reported for each year following index date (adjusted for competing risk of death with 95\% CI) and mean cumulative count (MCC) (with 95\% CI) of the number of subsequent exacerbations, for each year following index date and adjusted for competing risk of death were also calculated. Median time (in days) to exacerbation (as cumulative risk), adjusted for competing risk of death according to Fine and Gray ${ }^{19}$ was reported for each baseline exacerbation category.

\section{Healthcare Resource Utilization}

Since observation times of patients included differed, visit rates were calculated and reported as the number of visits that occurred per 100 patient years (PY) of total patient observation time, to account for both the number of patients included in the study, as well as the amount of time each patient could be observed in the study. Therefore, visit rates reflect the average number of visits per 100 (observed) patient years. Visit rates per 100 PY during baseline and by baseline exacerbation group during follow-up for COPD and all other visit reasons were evaluated for general practitioner (GP), outpatient hospital, hospitalization, and emergency room visits. Since outpatient hospital and GP visit rates in the database reflect a contact with a certain clinic/facility per calendar year quarter, the visit rate reflects the number of contacts per clinic, per $100 \mathrm{PY}$.

\section{Results}

\section{Baseline Data (Demographics, Treatment Groups, Healthcare Resource Utilization Comorbidities)}

Of the approximately 4.5 million patients in the WIG2 database, 250,723 patients with COPD met the inclusion criteria, with a total of over 1.2 million PY of data, and a mean of 5.04 and range of $1-8$ follow-up years. Just over half of patients were male $(57.5 \%)$ and patients were on average 63 years (mean age) (Table 1). Figure 1 shows the study flow from the WIG2 database to the final study population. Patients were categorized into how many exacerbations they experienced in the 12-month baseline period, prior to index date.

The mean follow-up period by treatment group was comparable, ranging from $4.72-5.50$ years (see Table S2). Of all patients, $78 \%$ had experienced no exacerbations during the baseline period, with the rest evenly distributed: $11 \%$ had 1 moderate exacerbation (B) and $11 \%$ had either 1 severe or multiple exacerbations (C) (Figure 1). Patients in category $\mathrm{C}$ had an exacerbation rate of $3.00(95 \% \mathrm{CI}$ 2.98-3.02) in the baseline year.

Of all patients meeting inclusion criteria, most (65.4\%) had none of the guideline-recommended inhaled maintenance treatments in the 12 months before index. Most (70.9\%) of the patients without prior exacerbations (category 
Table I Baseline Demographic Information, Follow-Up Time, and Baseline Exacerbations

\begin{tabular}{|l|l|}
\hline Patients, Total & $\mathbf{2 5 0 , 7 2 3}$ \\
\hline Age, mean (range) & $63.0(40-107)$ \\
\hline Male & $57.5 \%$ \\
\hline Total person years follow-up time & $1,264,265.0$ \\
\hline Follow-up, mean & 5.04 \\
\hline Follow-up, median & 5 \\
\hline Pneumonia & $10,667(4.3 \%)$ \\
\hline Asthma & $52,608(21 \%)$ \\
\hline Osteoporosis & $2169(8.7 \%)$ \\
\hline Diabetes & $29,728(11.9 \%)$ \\
\hline Ischaemic heart disease & $54,335(21.7 \%)$ \\
\hline PAD & $15,115(6.0 \%)$ \\
\hline Stroke & $8490(3.4 \%)$ \\
\hline Atrial fibrillation & $21,842(8.7 \%)$ \\
\hline Dementia & $8215(3.3 \%)$ \\
\hline Heart failure & $32,598(13.0 \%)$ \\
\hline Malignant neoplasm & $29,998(12.0 \%)$ \\
\hline Depression and Anxiety & $53,144(21.2 \%)$ \\
\hline Number of baseline & Exacerbation rate per I00 PY \\
\hline exacerbations & $(95 \%$ Cl) \\
\hline Category A & 0 \\
\hline Category B & \\
\hline Category C & $(2.98-3.03)$ \\
\hline
\end{tabular}

A), just over half with one moderate baseline exacerbation (category B), and over a third (36.9\%) of patients with one severe baseline or multiple baseline exacerbations (category C), were not taking any LABA, LAMA or ICS inhaled treatment in the 12 months prior to index. A combination of ICS with LABA or LAMA (16.4\%) was the most frequently used treatment option. Patients were least likely to be treated with a LABA/LAMA combination therapy $(1.7 \%)$. Patients in category $\mathrm{C}$ were about as likely to be taking a triple combination LABA, LAMA and ICS therapy $(22.4 \%)$, as a LABA or LAMA in combination with ICS $(24.8 \%) ; 4.1 \%$ and $4.6 \%$ were being treated with a LABA plus LAMA combination therapy, or ICS monotherapy, respectively (see Figures $\mathrm{S} 1$ and $\underline{\mathrm{S} 2}$ ).
Outpatient visits for other reasons were by far the most used resource, whereas GP visits due to other reasons were the second most used resource in nearly all baseline treatment groups (Figure 2).

At baseline, ischaemic heart disease $(21.7 \%)$, depression and/or anxiety $(21.2 \%)$, and asthma (21\%) were the most frequently observed comorbidities. Other cardiovascular-related comorbidities investigated were heart failure, atrial fibrillation, peripheral artery disease, and stroke, seen in $13.0 \%, 8.7 \% 6.0 \%$ and $3.4 \%$ of patients during baseline, respectively (Table 1). More than half (65\%) of the patients with an asthma diagnosis were taking either one of the treatment regimens that included an ICS (see Table S3,Figure S3).

\section{Subsequent Exacerbations: Cumulative Incidence and Mean Cumulative Count (MCC)}

Most (81.7\%; 95\% CI 81.2-82.2) of category C patients had experienced a subsequent exacerbation after three years of follow-up, whereas 59.1\% (95\% CI 58.5-59.7) of patients in category B, and 35.9\% (95\% CI 35.7-36.1) of patients in category $\mathrm{A}$, had a subsequent exacerbation during that period (Figure 3, and Table S4). By 8 years, 87.0\% (86.6-87.4), 70.5\% (69.9-71.0) and 49.1\% (48.949.3) of category C, B and A patients (respectively) had experienced a subsequent exacerbation.

The average cumulative number of subsequent exacerbations in category $\mathrm{C}$ patients was 6.26 (95\% CI 6.176.34) after three years of follow-up. In categories B and A, the cumulative average at three years was 1.89 (95\% CI 1.85-1.92) and 0.89 (95\% CI 0.88-0.90), respectively. By the end of the 8-year follow-up period, the difference seen between the different categories widened, with the most substantial increase seen in category $\mathrm{C}$ patients; this group experienced a mean of 14.05 (95\% CI 13.84-14.26) exacerbations, compared with 4.70 (95\% CI 4.61-4.80) and 2.46 (95\% CI 2.44-2.49) in categories B and A, respectively (Figure 4, and Table S5).

\section{Time Until Subsequent Exacerbation}

On average, it took only 36 days (95\% CI 35-37) after index date for $25 \%$ of the patients in category $\mathrm{C}$ to experience an exacerbation; category $\mathrm{B}$ patients reached this quartile in 166 days (95\% CI 160-173) and category A patients in 569 days (95\% CI 561-577). It took an average of 579 days (95\% CI 553-611), for $75 \%$ of the patients in category 


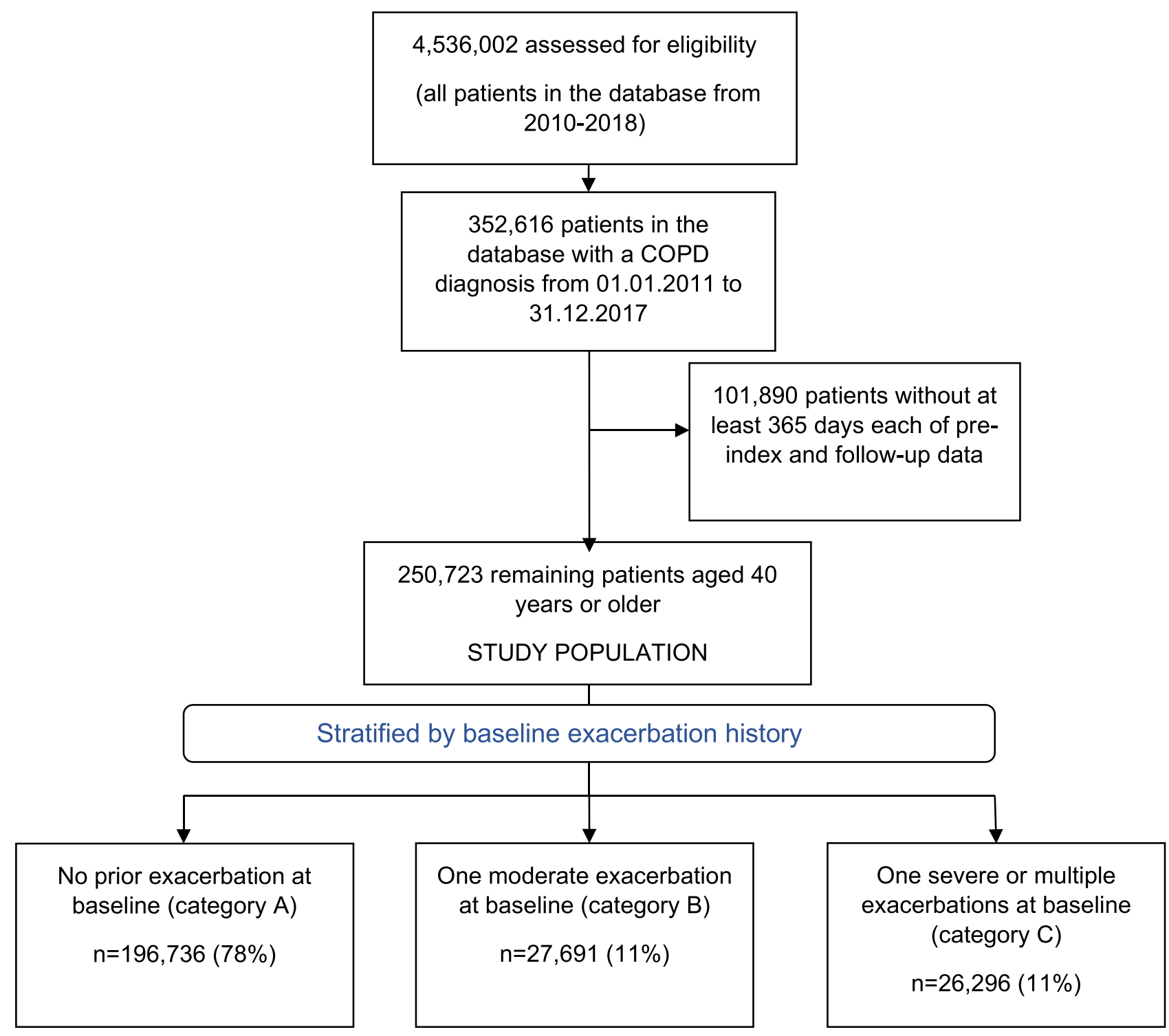

Figure I Study population selection and baseline exacerbation history.

C to have a subsequent exacerbation, whereas it took category B patients 2474 days $(95 \%$ CI $2371-2579)$ to reach this quartile. Category A patients did not reach the $75 \%$ quartile during observation (see Table S6).

\section{Risk of a First Subsequent Exacerbation During Follow-Up}

Both category $\mathrm{B}$ and $\mathrm{C}$ patients had a higher risk (using hazard ratio risk estimates) of a first moderate exacerbation in the follow-up period (hazard ratio $1.89,95 \% \mathrm{CI}$ $1.87-1.90$ and $3.93,3.91-3.94$, respectively) when compared with category A patients. The hazard ratio for a first severe exacerbation was $1.30(1.27-1.34)$ for category
B patients and 2.96 (2.94-2.99) for category C patients (Figure 5, Supplemental Material Table S6).

\section{Exacerbation Effect on Follow-Up HCRU}

During follow-up, healthcare visits were reported per 100 PY, to account for the different length of follow-up of different patients (Table 2). Patients were only required to have 12-months of follow-up data to be included in the study; however, they were followed for as long as data were available. We added up all the follow-up time of all patients and reported the visits as a function of the total PYs (and then per $100 \mathrm{PY)}$ of data available. As an example, this translates to there being 234.03 COPD- 


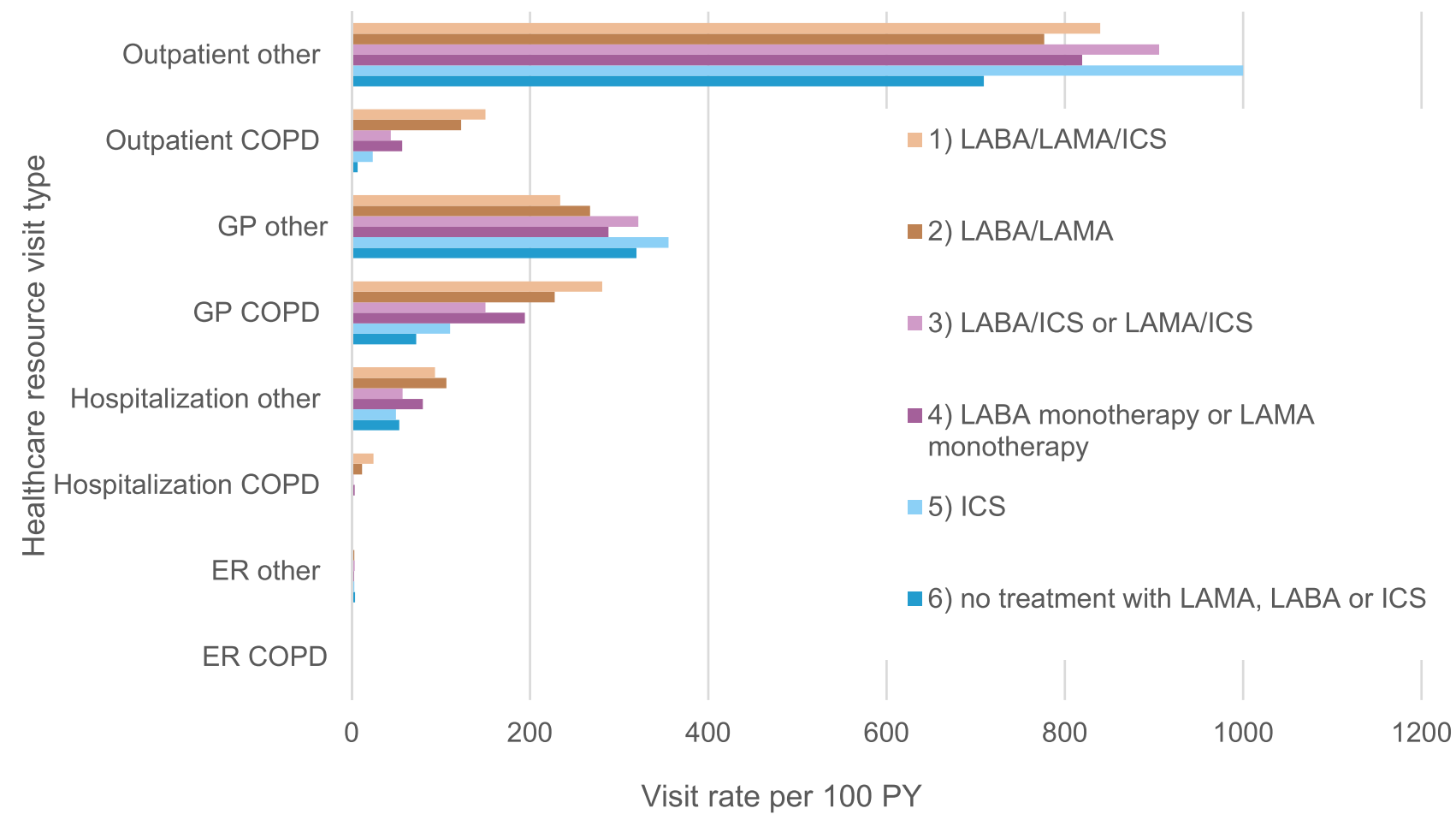

Figure 2 Healthcare resource utilization visit rate by baseline treatment group and visit type and reason, per 100 PY. Abbreviations: LABA, long-acting $\beta 2$-agonist; LAMA, long-acting muscarinic antagonist; ICS, inhaled corticosteroid.

\section{Cumulative incidence of a subsequent exacerbation}

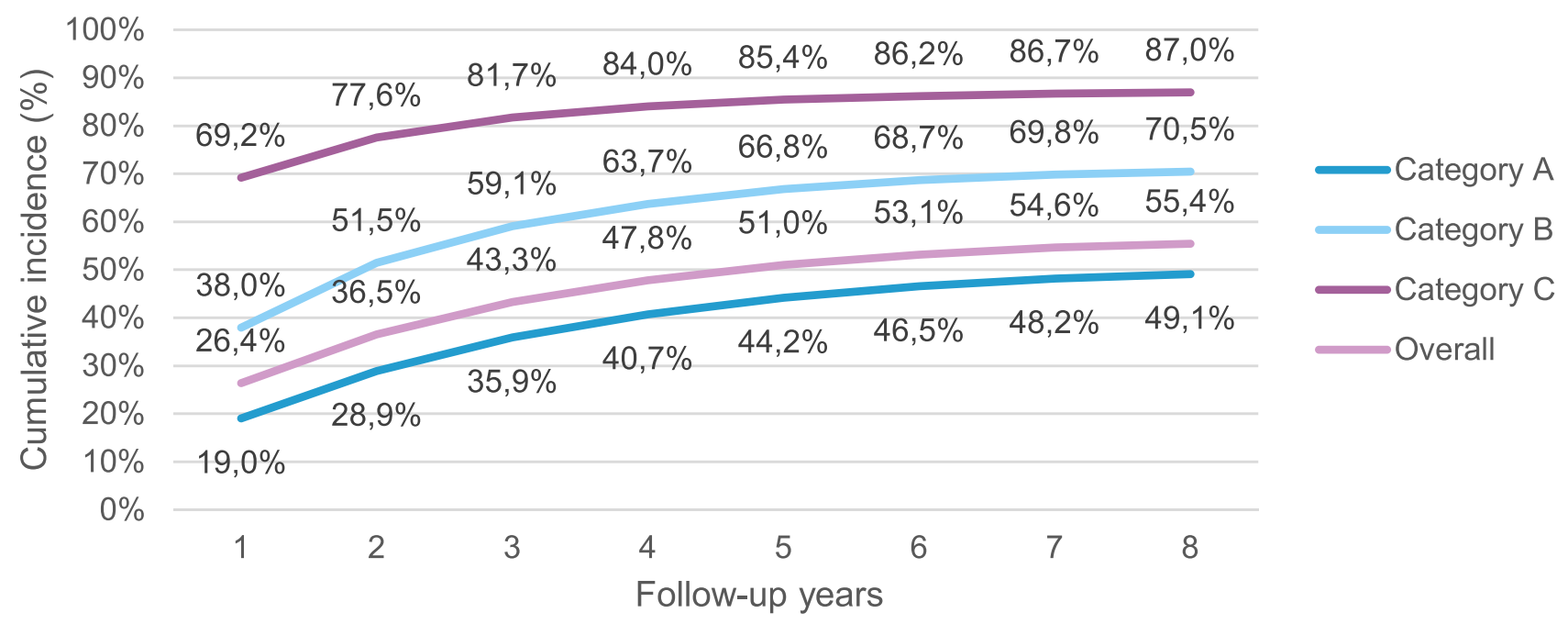

Figure 3 Cumulative incidence of a subsequent exacerbation, adjusted for competing risk of death, by time since index date. (A) No exacerbations at baseline, (B) one moderate exacerbation, (C) one severe or multiple (regardless of severity) exacerbation at baseline. $95 \% \mathrm{Cl}$ available in Supplemental Material Table S4.

related GP visits per 100 category A patients each year. The most used resource was other non-COPD related outpatient visits, and the second most used resource overall being GP visits for COPD. Category A patients, despite being exacerbation-free in the baseline year, had nearly as many visits to their GP due to their COPD disease as category B patients. Non-COPD hospitalization rates were higher than hospitalizations for COPD across all categories; however, hospitalization among category C patients was substantially higher (for both COPD and non-COPD related visits) than all other categories. Category $\mathrm{C}$ patient visit rates were highest for nearly all 


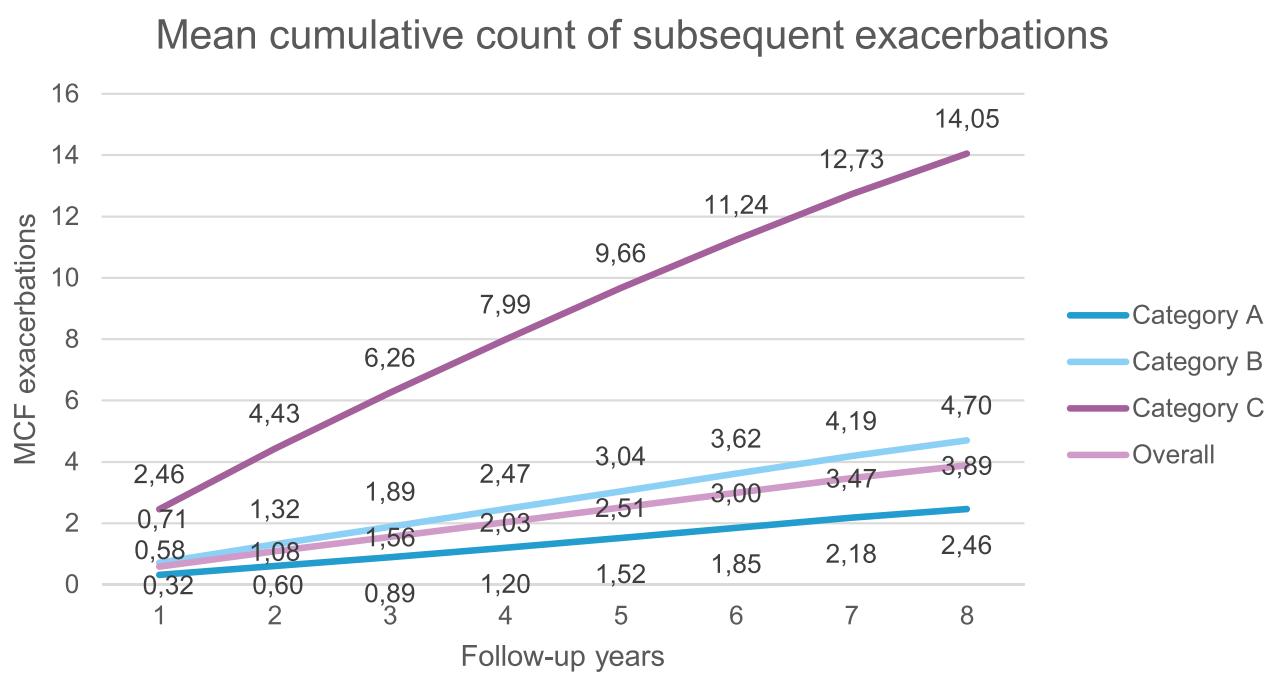

Figure 4 Mean cumulative count (MCC) of a subsequent exacerbation in the overall disease population, by time since index date, adjusted for competing risk of death. (A) No exacerbations at baseline, (B) one moderate exacerbation, (C) one severe or multiple (regardless of severity) exacerbation at baseline. $95 \% \mathrm{Cl}$ available in Supplemental Material Table S5.

\section{Risk of a future exacerbation}

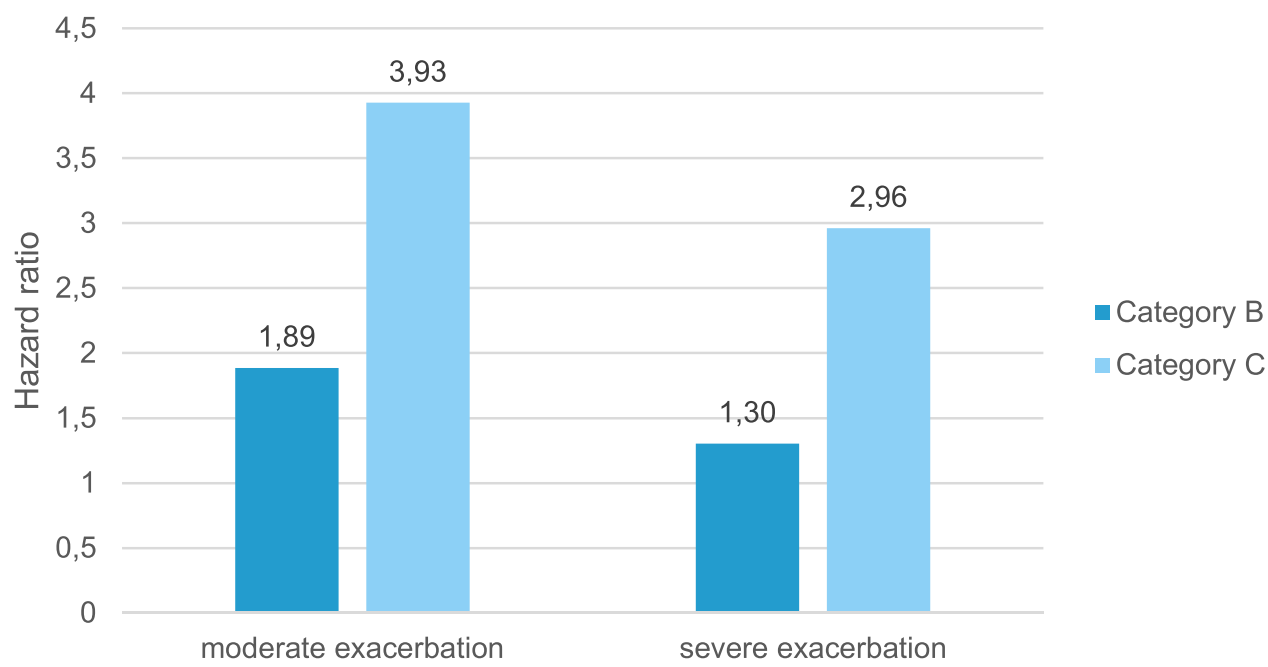

Figure 5 Risk of a future exacerbation compared to category A (reference) during follow-up by baseline exacerbation category. (B) One moderate exacerbation, and (C) one severe or multiple (regardless of severity) exacerbation at baseline. $95 \% \mathrm{Cl}$ available in Supplemental Material Table S7.

healthcare visit types (for both COPD and other), followed by category $\mathrm{B}$ patients and finally category A patients.

\section{Discussion}

Our study provides up to 8 years of longitudinal data from over 250,000 patients in Germany. We included all patients with a COPD diagnosis, excluding only patients with insufficient data and those under 40 years of age. Patient health data was collected over an average of 5.04 years. Our population was mostly
(78\%) exacerbation-free in the 12 months prior reflecting a COPD population with potentially many newly diagnosed cases, with a comparable proportion of patients having severe/multiple events or single moderate exacerbations at baseline. 149,290 out of our entire population $(59.5 \%)$ had no documented COPD diagnosis in the 12 months prior to their index date, meaning they were potentially incident COPD patients. The exacerbation rate in the 12 months prior to inclusion (during baseline) for patients with multiple or 
Table 2 Healthcare Resource Utilization Visit Rate During Follow-Up by Visit Type and Reason, per 100 PY. (A) No Exacerbations at Baseline, (B) One Moderate Exacerbation, (C) One Severe or Multiple (Regardless of Severity) Exacerbation at Baseline

\begin{tabular}{|c|c|c|c|c|}
\hline \multirow[t]{2}{*}{ HCRU Visit Type (per 100 PY with 95\% CI) } & \multicolumn{3}{|c|}{ Baseline Exacerbation Category } & \multirow[t]{2}{*}{ Overall } \\
\hline & A & B & C & \\
\hline GP visit rate COPD & $234.03(233.28-234.78)$ & $233.00(230.94-235.06)$ & $270.79(268.46-273.12)$ & $237.77(237.09-238.45)$ \\
\hline GP visit rate other non-COPD & $228.18(227.08-229.28)$ & $259.55(256.50-262.60)$ & $253.94(250.52-257.35)$ & $234.34(233.35-235.34)$ \\
\hline Outpatient visit rate COPD & $85.19(84.56-85.83)$ & $105.97(104.06-107.87)$ & 141.93 (|39.55-|44.32) & $93.44(92.84-94.04)$ \\
\hline Outpatient visit rate other non-COPD & $826.53(822.82-830.24)$ & $1021.64(1010.39-1032.88)$ & $1085.60(1073.19-1098.01)$ & $875.25(87 I .8 I-878.70)$ \\
\hline Hospitalization rate COPD & $2.98(2.89-3.07)$ & $4.04(3.73-4.34)$ & $18.32(17.52-19.12)$ & $4.70(4.59-4.82)$ \\
\hline Hospitalization rate other non-COPD & $67.00(66.37-67.63)$ & 75.97 (74.23-77.72) & 109.25 (107.1I-1 II.39) & $72.43(71.85-73.00)$ \\
\hline ER visit rate COPD & $0.05(0.04-0.07)$ & $0.07(0.03-0.10)$ & $0.11(0.06-0.16)$ & $0.06(0.05-0.07)$ \\
\hline ER visit rate other non-COPD & $4.98(4.8 I-5.14)$ & $6.14(5.64-6.64)$ & $7.02(6.39-7.66)$ & $5.32(5.16-5.47)$ \\
\hline
\end{tabular}

severe exacerbations was 3.00 (95\% CI 2.98-3.02). As reported in previous studies, we also found that multiple previous exacerbations result in a greater likelihood of subsequent exacerbations..$^{9,11,12,20}$

\section{Inhaled Maintenance Treatment}

Most (65.4\%) patients in our study were using none of the inhaled maintenance treatments LABA, LAMA or ICS at baseline. This may be related to the following: $78.5 \%$ of our population were without exacerbations in the year prior, and our population may contain a substantial proportion of incident patients; and our study only looked at the use of LAMA, LABA and ICS inhaled treatments, so any other treatments to manage symptoms, like short-acting bronchodilators, would not have been captured. Altogether, around $27 \%$ of all patients were taking an ICS, most of them as part of a combination treatment with LABA and/or LAMA. Surprisingly, over a third of patients in category $\mathrm{C}$ had none of the inhaled maintenance treatments we investigated during the baseline year, particularly since the most recent GOLD document, ${ }^{2}$ as well as those documents applicable to our study observation period, ${ }^{21,22}$ recommend initiating a long-acting bronchodilator treatment (either mono LAMA or LAMA/ LABA) with or without an ICS, prior to discharge from hospital following a severe exacerbation.

We stratified patients by their baseline treatment group and looked at healthcare resource utilization during baseline. Hospitalization (both for COPD and other non-COPD reasons) occurred more for patients on a LABA/LAMA/ICS triple combination therapy or patients taking a LABA/
LAMA combination therapy at baseline, but this likely reflects their underlying disease severity. Patients with baseline treatment use in these two categories also had the highest percentages of baseline comorbidities, including pneumonia, ischemic heart disease, atrial fibrillation, heart failure, and malignant neoplasms. This may reflect the underlying complexity of these patients, and the need for good COPD disease management, allowing for better management of these comorbidities. In fact, one-third of patients in each of these baseline treatment groups had ischemic heart disease during baseline, and one quarter had heart failure.

\section{Exacerbations}

Patients with multiple baseline exacerbations experienced subsequent exacerbations substantially earlier and more often than those with just a single baseline exacerbation. Of all patients studied, $26.4 \%$ experienced a subsequent exacerbation after one year of follow-up, compared to a total of $22 \%$ (both categories B and C) during the baseline year. This still leaves nearly $75 \%$ of patients overall that were exacerbation free after the first year of follow-up; however, we see that the risk of exacerbation does not disappear; after 8 years, another $25 \%$ had experienced an exacerbation. This should have implications on treatment, reminding healthcare providers of the importance of continuous exacerbation prevention: in a given year patients may not exacerbate but still have a significant risk of experiencing subsequent events in the future.

In another observational database study $(15,857$ patients, average follow-up 2.4 years) patients with a severe (defined by hospitalization) exacerbation as the 
index event were stratified into three groups of one severe, one moderate or no exacerbation at baseline, respectively, prior to inclusion. ${ }^{12}$ In this more severe population, most similar to our category $\mathrm{C}$ population, the group most likely to experience a subsequent exacerbation was the group with a severe exacerbation at baseline; the probability of having a subsequent exacerbation was $75 \%$ at one year from index event in the entire population, increasing to $92 \%$ after three years. In our study, the cumulative incidence was similar for our category $\mathrm{C}$ population, with $69 \%$ at 1 year and $82 \%$ after three years.

The mean number of exacerbations was highest at each follow-up timepoint for patients in category $\mathrm{C}$ and after 8 years of follow-up, the average number of exacerbations in category $\mathrm{C}$ patients was three times higher than in category B patients, and 5.7 times higher than in category A patients. Additionally, category $\mathrm{C}$ patients showed a much faster rate of increase over the 8 years than the other groups. In contrast, Blasi et $\mathrm{al}^{12}(15,857$ patients with a severe exacerbation, comparable to category $\mathrm{C}$ in our study) found slightly higher exacerbation rates per PY (3.2 per PY) than in this study, while other reports range from about 0.5 to 3.5 annual exacerbations per patient. ${ }^{23}$ Both category $\mathrm{B}$ and $\mathrm{C}$ patients were more at risk of a future moderate than a severe exacerbation, but risk levels for both exacerbation types were more than twice as high for category $\mathrm{C}$ than $\mathrm{B}$ patients. However, having had even a single moderate baseline exacerbation increased the risk of subsequent moderate exacerbations by a factor of almost $2(1.89,95 \%$ CI $1.87-1.90)$, and that of a severe exacerbation by $30 \%$. This further reinforces the importance of treatment and attention to disease management following even a moderate exacerbation.

In our study, $80 \%$ of category A patients (no exacerbation during baseline year) experienced no exacerbation within the first year of follow-up, similar to findings of $75 \%$ remaining exacerbation free from previous studies. ${ }^{11}$ After 8 years of follow-up, nearly half (49.1\%) of category A patients had exacerbated. ${ }^{20}$ These findings emphasize that patient care even in patients without exacerbations should be monitored closely.

\section{Healthcare Resource Utilization}

A history of severe or multiple exacerbations is associated with increased use of healthcare resources. While overall utilization and presumably costs were highest for patients in category $\mathrm{C}$, some visit types (especially visits to a GP) were similar in category A patients. We did see a substantially higher rate of hospitalization (a known cost-driver in healthcare) in patients with multiple or severe baseline exacerbations. Another study looked at healthcare use and costs in a US population (almost 9000 patients aged 40-89) using claims data and found incrementally higher costs in patients with more exacerbations. $^{24}$ This is similar to what Wacker et $\mathrm{al}^{25}$ found in a sample of nearly 2500 patients in Germany aged 40 years and older; physician visits were increased by a factor of 2 compared with non-COPD controls, regardless of COPD disease grade (defined in this study by $\mathrm{FEV} 1 \%$ predictive values rather than exacerbations). Hospitalization and prescribed medications were however increased in higher grades of disease than lower, as we observed in our study as well. While we did not compare patients without COPD, studies have shown that even at mild disease grades, having COPD is associated with higher direct and indirect healthcare costs, compared to lung-healthy control subjects, even when adjustments for major comorbidities were made. ${ }^{24,25}$

\section{Study Limitations}

Our study has some limitations: the data generated during standard clinic visits was not collected with research purposes in mind; some data may be missing or incorrectly coded. Although typical for observational studies, we were unable to complete any missing or incomplete data, as would be possible in the controlled environment of a clinical study; therefore, patients without continuous follow-up data for any reason (changed SHI provider, data documented poorly by healthcare providers) within the 12-months follow-up were not included in the dataset. However, if any of these occurred after the 12-months of follow-up, the patient would have nonetheless been included, and we expect this effect to be minor. Specific to the indication, we used a diagnosis code to confirm diagnosis and data on treatment or hospitalization to evaluate disease severity, without an actual clinical assessment or data on spirometry to confirm; to determine a moderate exacerbation, we used data on antibiotics or OCS as criteria, which could have been given for other reasons than an exacerbation. If an exacerbation was treated in an outpatient setting with intravenous corticosteroids, we would not have been able to evaluate this moderate exacerbation, as only treatment with oral corticosteroids was captured. We also included patients diagnosed with other comorbidities for which the differential diagnosis of COPD may be 
challenging, such as asthma; ${ }^{2}$ these factors may skew our results in either way.

Our study includes patients with a COPD diagnosis occurring anywhere from 2011-2017; due to the study design, this may result in a higher proportion of incident disease patients being included from 2012 onwards. This may influence the make-up of our baseline exacerbation groups and study results.

There has been some evidence showing that some mild exacerbations not reported by patients to healthcare providers (and remaining untreated), still have an influence on the medium-term course of the disease. ${ }^{26}$ These patients could have been included in the wrong baseline exacerbation group in our study, since our data could only provide exacerbation events for which there was healthcare utilization. On the other hand, any documented lower respiratory tract infections treated in hospital that were actually presentations of a COPD exacerbation, may not have been documented as such. In this case, the actual proportion of patients in category $\mathrm{C}$ may have been underestimated, had these been documented as COPD hospitalization, and therefore a serious exacerbation.

While our study provides a complete picture of the medications taken by patients in the baseline year, the design did not include medications throughout the 8-year follow-up. Buhl et $\mathrm{al}^{11}$ however looked at persistence to medication regimes in around 6000 German patients and found that $77.2 \%$ of patients with baseline medication data were still receiving the same class of medications at the 1-year follow-up visit. This may be transferable to our data on exacerbations after 1 year of follow-up, however including data on treatment throughout the 8 years followup could provide valuable information and further improve treatment guidelines.

\section{Conclusions}

This study used a very large real-world population with up to 8 years of follow-up data and without selection or participation bias, uncovering real-world outcomes sometimes obscured in randomized control trials (RCTs). While selective inclusion criteria of RCTs provide strong evidence for internal validity of therapeutic outcomes, our study provides strong external validity of results.

Having had multiple and/or severe exacerbations increases the likelihood of, and reduces time to, a subsequent exacerbation, and also leads to an increased rate in number of exacerbations over time. In addition, multiple exacerbations reduce the time to the next exacerbation. Since having even a single moderate exacerbation results in an increased risk of subsequent exacerbations, and with a considerable number of patients without exacerbations during the baseline year experiencing an exacerbation during follow-up, maintenance treatments that reduce the risk of future exacerbations should be prescribed.

\section{Data Sharing Statement}

The database used in this study cannot be made publicly available due to data protection laws in Germany. To enable replication of results, the anonymized data used for this study are stored as backup on a secured drive.

\section{Acknowledgments}

Financial support of the study was provided by AstraZeneca. Some of these data have been previously presented in conference abstract form.

\section{Funding}

Funding for this study was provided by AstraZeneca.

\section{Disclosure}

JD and NK are both employees of WIG2 GmbH. MP is an employee of Kantar GmbH and FWF is employed by AstraZeneca. CFV reports grants and/or personal fees from AstraZeneca, Boehringer Ingelheim, CSL Behring, Chiesi, GlaxoSmithKline, Grifols, Menarini, Novartis, Nuvaira, MedUpdate, and Aerogen, outside the submitted work. The authors report no other conflicts of interest in this work.

\section{References}

1. World Health Organization. The top 10 causes of death.Available from: https://www.who.int/news-room/fact-sheets/detail/the-top-10causes-of-death. Published December 9 2020. Accessed April 24, 2021.

2. Global Initiative for Chronic Obstructive Lung Disease. Global strategy for the diagnosis, management and prevention of chronic obstructive pulmonary disease (2021 Report); 2021. Available from: https:// goldcopd.org/wp-content/uploads/2020/11/GOLD-REPORT-2021-v1. 1-25Nov20_WMV.pdf. Accessed February 5, 2021.

3. Han MK, Agusti A, Celli BR, et al. From global initiative for chronic obstructive lung disease stage 0 to pre-chronic obstructive pulmonary disease. Am J Resp Crit Care Med. 2021;203(4):414-423.

4. Gibson GJ, Loddenkemper R, Sibille Y, et al. The European Lung White Book. Respiratory health and disease in Europe: Ch 2 the economic burden of lung disease. Available from: https://www.erswhi tebook.org/files/public/Chapters/02_economics.pdf. Accessed December 4, 2020.

5. Celli BR, MacNee W. Standards for the diagnosis and treatment of patients with COPD: a summary of the ATS/ERS position paper. Eur Respir J. 2004;23(6):932-946. doi:10.1183/09031936.04.00014304 
6. Ritchie AI, Wedzicha JA. Definition, causes, pathogenesis, and consequences of chronic obstructive pulmonary disease exacerbations. Clin Chest Med. 2020;41(3):421-438. doi:10.1016/j. ccm.2020.06.007

7. Cardoso J, Coelho R, Rocha C, Coelho C, Semedo L, Bugalho Almeida A. Prediction of severe exacerbations and mortality in COPD: the role of exacerbation history and inspiratory capacity/ total lung capacity ratio. Int $J$ Chron Obstruct Pulmon Dis. 2018;13:1105-1113. doi:10.2147/COPD.S155848

8. López-Campos JL, Tan W, Soriano JB. Global burden of COPD. Respirology. 2016;21(1):14-23. doi:10.1111/resp.12660

9. Hoogendoorn M, Feenstra TL, Boland M, et al. Prediction models for exacerbations in different COPD patient populations: comparing results of five large data sources. Int J Chron Obstruct Pulmon Dis. 2017;12:3183-3194. doi:10.2147/COPD.S142378

10. Kerkhof M, Freeman D, Jones R, Chisholm A, Price DB. Predicting frequent COPD exacerbations using primary care data. Int J Chron Obstruct Pulmon Dis. 2015;10:2439-2450. doi:10.2147/COPD. S94259

11. Buhl R, Criée C-P, Kardos P, et al. A year in the life of German patients with COPD: the DACCORD observational study: two cohorts, observational, comparing patients on a treatment including LAMA or one not including LAMA. No intervention. 6000 patients in Germany, at 500 clinics. Int J Chron Obstruct Pulmon Dis. 2016;11:1639-1646. doi:10.2147/COPD.S112110

12. Blasi F, Cesana G, Conti S, et al. The clinical and economic impact of exacerbations of chronic obstructive pulmonary disease: a cohort of hospitalized patients. PLoS One. 2014;9(6):e101228. doi:10.1371/ journal.pone.0101228

13. Vogelmeier C, Nuevo J, Telg G, et al. Real-life treatment patterns and burden of disease among patients with COPD - a large observational study. In proceedings of B41. therapeutic intervention, quality improvement, and treatment adherence in obstructive lung disease. American Thoracic Society; May, 2020:A3331-A3331.

14. Vogelmeier CF, Diesing J, Kossack N, Pignot M, Friedrich F. Risk of future exacerbations among COPD patients - a real-world registerbased cohort study in Germany. Abstracts zum 127. Kongress der Deutschen Gesellschaft für Innere Medizin e.V [Congress of the German Society for Internal Medicine e.V]. Internist (Berl). 2021;62(Supp12):147-202. doi:10.1007/s00108-021-01036-7

15. Vogelmeier C, Pignot M, Wiklund F, Nuevo J, Telg G, Janson C. Risk of future exacerbations among COPD patients - a real-world registerbased cohort study (AvoidEx). Eur. Resp. J. 2020;09072020:4189. doi:10.1183/13993003.congress-2020.4189

16. Vogelmeier CF, Diesing J, Kossack N, Pignot M, Friedrich F. P631 risk of future exacerbations among COPD patients- a real-world register-based cohort study in Germany. In proceedings of 61 . Kongresses der Deutschen Gesellschaft für Pneumologie und Beatmungsmedizin e. V. Latebreaking Abstracts. Pneumologie. 2020;74(S01):S147. doi:10.1055/s-0037-1599663
17. Birkeland KI, Bodegard J, Banerjee A, et al. Lower cardiorenal risk with sodium-glucose cotransporter-2 inhibitors versus dipeptidyl peptidase- 4 inhibitors in patients with type 2 diabetes without cardiovascular and renal diseases: a large multinational observational study. Diabetes Obes Metab. 2021;23(1):75-85. doi:10.1111/ dom.14189

18. Dong H, Robison LL, Leisenring WM, Martin LJ, Armstrong GT, Yasui Y. Estimating the burden of recurrent events in the presence of competing risks: the method of mean cumulative count. Am J Epidemiol. 2015;181(7):532-540. doi:10.1093/aje/kwu289

19. Fine JP, Gray RJ. A proportional hazards model for the subdistribution of a competing risk. J Am Stat Assoc. 1999;94(446):496-509. doi:10.1080/01621459.1999.10474144

20. Vogelmeier CF, Kostikas K, Fang J, et al. Evaluation of exacerbations and blood eosinophils in UK and US COPD populations. Respir Res. 2019;20(1):178. doi:10.1186/s12931-019-1130-y

21. Rabe KF, Hurd S, Anzueto A, et al. Global strategy for the diagnosis, management, and prevention of chronic obstructive pulmonary disease: GOLD executive summary. Am J Respir Crit Care Med. 2007;176(6):532-555. doi:10.1164/rccm.200703-456SO

22. Vestbo J, Hurd SS, Agustí AG, et al. Global strategy for the diagnosis, management, and prevention of chronic obstructive pulmonary disease: GOLD executive summary. Am J Respir Crit Care Med. 2013;187(4):347-365. doi:10.1164/rccm.201204-0596PP

23. Seemungal TA, Hurst JR, Wedzicha JA. Exacerbation rate, health status and mortality in COPD - a review of potential interventions. Int J Chron Obstruct Pulmon Dis. 2009;4:203. doi:10.2147/copd. s3385

24. Pasquale MK, Sun SX, Song F, Hartnett HJ, Stemkowski SA. Impact of exacerbations on health care cost and resource utilization in chronic obstructive pulmonary disease patients with chronic bronchitis from a predominantly Medicare population. Int J Chron Obstruct Pulmon Dis. 2012;7:757-764. doi:10.2147/COPD.S36997

25. Wacker ME, Jörres RA, Schulz H, et al. Direct and indirect costs of COPD and its comorbidities: results from the German COSYCONET study. Respir Med. 2016;111:39-46. doi:10.1016/j.rmed.2015.12.001

26. Jones PW, Lamarca R, Chuecos F, et al. Characterisation and impact of reported and unreported exacerbations: results from ATTAIN. Eur Respir J. 2014;44(5):1156-1165. doi:10.1183/09031936.00038814

\section{Publish your work in this journal}

The International Journal of COPD is an international, peer-reviewed journal of therapeutics and pharmacology focusing on concise rapid reporting of clinical studies and reviews in COPD. Special focus is given to the pathophysiological processes underlying the disease, intervention programs, patient focused education, and self management protocols. This journal is indexed on PubMed Central, MedLine and CAS. The manuscript management system is completely online and includes a very quick and fair peer-review system, which is all easy to use. Visit http://www.dovepress.com/testimonials.php to read real quotes from published authors. 